\title{
Effect of Thermal Processing on Anthocyanin Degradation in Two Bilberry Jam Formulations
}

\author{
MILENA NIKOLIC, ALEKSANDRA PAVLOVIC*, MILAN MITIC, SNEZANA MITIC, \\ SNEZANA TOSIC, JELENA MRMOSANIN, EMILIJA PECEV-MARINKOVIC \\ University of Nis, Faculty of Sciences and Mathematics, Department of Chemistry, Višegradska 33, P. O. Box 224, 18000 \\ Nis, Serbia
}

\begin{abstract}
Fresh bilberries were processed into sugar and sugar-low jams at high temperatures (90, 95, 100 and $105^{\circ} \mathrm{C}$ ) in order to follow degradation of total and individual anthocyanins. The greatest retention of all examined compounds was observed in sugar-low jam prepared at $90{ }^{\circ} \mathrm{C}$ for 5 minutes, while the greatest loss was detected in sugar jam prepared at $105^{\circ} \mathrm{C}$ for $30 \mathrm{~min}$. Cyanidin-3-galactoside and cyanidin-3-glucoside were found to be the most stable, while delphinidin-3-arabinoside and petunidin-3-arabinoside were the least stable.
\end{abstract}

Keywords: anthocyanins, antioxidant activity, bilberry, degradation kinetics

\section{Introduction}

Anthocyanins are water-soluble pigments which are responsible for blue, violet, pink and red colors of fruits of some plants [1]. They are found in strawberries [2], bilberries, blackcurrants and cowberries [3], raspberries and blackberries [4, 5]. Anthocyanins represent potent natural antioxidants with cardioprotective, anti-inflammatory and anticancer properties [5]. Bilberries (Vaccinium myrtillus) are one of the richest natural sources of anthocyanins [6] where they comprise up to $90 \%$ of total phenolic compounds [3]. Antioxidant activity of bilberries has been evaluated in several studies [7-9] and has been given in review works of Heinonen [10] and Szajdek and Borowska [11]. Therefore, bilberries are considered as functional food, with a beneficial effect on human health [11]. However, because of the seasonal character of bilberry fruits, it is important to determinate whether thermally processed forms, such as jams, could also represent a good source of nutrients.

Thermal degradation kinetics of anthocyanins follows first-order kinetics, where the rate constant increases with an increase in temperature [12]. Several published studies agree with previously mentioned [13-15].

According to de Moura et al. [15], the low-sugar blackberry jam prepared at $95^{\circ} \mathrm{C}$, can be considered a source of anthocyanin compounds even after six months of storage at an average temperature of $10^{\circ} \mathrm{C}$, since $19 \%$ loss of total anthocyanin content has been reported. García-Viguera et al. [16] reported losses in total anthocyanin content up to $40 \%$ (comparing to fresh fruit) during red raspberry jam manufacturing, with the jam being heated at $92^{\circ} \mathrm{C}$. In a study of Howard et al. [17], retention of $79 \%$ of total anthocyanins in sugar and sugar-free blueberry jams, which were heated until reached a full boil and held for $1 \mathrm{~min}$ on $103-105^{\circ} \mathrm{C}$, has been observed.

But according to our knowledge, the influence of high temperatures, which are often applied during bilberry jam preparation, on the content of total and individual anthocyanins has not been researched comparatively. Therefore, the objective of this study was to examine the influence of high temperatures and sugar on total and individual anthocyanin content, as well as an examination of anthocyanin degradation kinetic. Also, total phenolic and flavonoid contents and antioxidant activity by DPPH assay were determined in sugar and sugar-low bilberry jams

\footnotetext{
*email: aleksandra.pavlovic@pmf.edu.rs
} 


\section{Materials and methods}

Trolox was bought from Acros Organics (Morris Plains, New Jersey, USA). Malvidin-3-O-glucoside chloride, delphinidin-3-O-glucoside chloride, gallic acid, (+)-catechin, DPPH (HPLC grade), saccharose and glucose were purchased from Sigma Aldrich (Steinheim, Germany). Cyanidin-3- $O$-glucoside chloride (HPLC grade) was from ChromaDex (Irvine, CA, USA). Folin Ciocalteu's phenol reagent, sodium hydroxide, sodium acetate, sodium nitrite, sodium carbonate, potassium chloride, aluminum chloride hexahydrate, and hydrochloric acid were purchased from Merck ${ }^{\circledR}$ (Darmstadt, Germany). Methanol (HPLC grade) and ethanol (96\% by vol.) were purchased from J.T. Baker (Deventer, The Netherlands). For preparation of all samples and standards purified water $(18 \mathrm{M} \Omega \mathrm{cm})$ was used (prepared by a MicroMed purification system (TKA Wasseraufbereitungssysteme GmbH, Niederelbert, Germany).

\section{Instruments}

The spectrophotometer Agilent 8453 UV/Vis (Agilent Technologies, Santa Clara, USA) was used for absorbance measurements. The spectra were recorded by using optical cuvettes of $1 \mathrm{~cm}$ optical path. The Hanna instrument pH-meter (Hanna Instruments, Smithfield, Rhode Island, USA) equipped with a glass electrode was used for the $\mathrm{pH}$ measurements. A model 1200 (Agilent Technologies, Santa Clara, USA) was used for HPLC analysis. The separation was performed in $\mathrm{C}_{18}$ Zorbax Eclipse XDB-C18 column, $5 \mu \mathrm{m}, 4.6 \times 150 \mathrm{~mm}$ (Agilent Technologies, Santa Clara, USA).

\section{Samples}

Bilberry samples were harvested in Southeastern Serbia (Vlasina region). The amount of berries collected for the analysis was about $500 \mathrm{~g}$. Before the analysis, the samples (whole fresh fruits) were stored in the fridge at $-18{ }^{\circ} \mathrm{C}$. Frozen bilberry fruits were milled in the blender to obtained puree which was subsequently used for jam preparation. Two formulations of jams (sugar and sugar-low) were prepared according to a slightly modified method described by de Moura et al. [15]. The sugar formulation implied $60 \%$ of fruit puree, $30 \%$ of saccharose, $9.8 \%$ of glucose and $0.2 \%$ of commercially available pectin for domestic use. The sugar-low formulation implied $94.2 \%$ of fruit puree, $5 \%$ of saccharose and $0.8 \%$ of commercially available pectin for domestic use.

\section{Heating procedure}

The mixtures were heated to temperatures of $90^{\circ} \mathrm{C}, 95^{\circ} \mathrm{C}, 100{ }^{\circ} \mathrm{C}$, and $105^{\circ} \mathrm{C}$ and frequently stirred in opened vessels. During the heat treatment, the temperature was registered using laboratory thermometer and stabilized at process temperature $\left( \pm 1^{\circ} \mathrm{C}\right)$. Once isothermal conditions were reached, samples were taken at different heating times: 0, 5, 10, 15, 20 and $30 \mathrm{~min}$. In order to stop further thermal degradation, the samples were immediately immersed into cold water. The analysis was done immediately.

\section{Extraction procedure}

The ultrasonic extractions of jam samples were performed at room temperature $\left(25^{\circ} \mathrm{C}\right)$ for $15 \mathrm{~min}$ three times. Amount of $5.0000 \mathrm{~g} \pm 0.0001 \mathrm{~g}$ of each sample was weighed, then was mixed with $5 \mathrm{~mL}$ acidified methanol with $\mathrm{HCl}(1 \%)$ and put into the ultrasonic bath and sonicated for $15 \mathrm{~min}$. All the extracts were filtered, and the clear supernatants were collected. Three times repeated extractions were made, the fractions were collected and evaporated to dryness by rotary evaporation under reduced pressure at $40^{\circ} \mathrm{C}$. Ultrapure water was added to $10 \mathrm{~mL}$ and these solutions were used for further analysis.

\section{HPLC analysis}

To identify and determine the individual anthocyanins content Agilent-1200 series HPLC with the $\mathrm{UV}-\mathrm{Vis}$ photodiode array detector (DAD) was used. The column was thermostated at $25^{\circ} \mathrm{C}$. After injecting $5 \mu \mathrm{L}$ of sample, the separation was performed in an Agilent-Eclipse XDB C-18 $4.6 \times 150 \mathrm{~mm}$ column. The mobile phase consisted of two solvents, which were used for gradient eluation: $5 \%$ formic 
acid, aqueous (eluent $\mathrm{A}$ ) and $80 \%$ acetonitrile/5\% formic acid/15\% purified water (eluent B). The applied elution program was described in detail by Mitić et al. [18]. Identifications of individual compounds were based on the retention times and spectral data with those of the standards or with data (petunidin-3-galactoside, peonidin-3-galactoside, and petunidin-3-arabinoside) reported in the literature $[19,20]$. Quantitative determination of individual anthocyanins in samples was calculated using calibration curves. Petunidin-3-galactoside, peonidin-3-galactoside, petunidin-3-arabinoside, cyaniding-3arabinoside, and cyaniding-3-galactoside were quantified using the calibration curve of cyanidin-3-Oglucoside [19]. Delphinidin-3-galactoside and delphinidin-3-arabinoside were quantified using the calibration curve of delphinidin-3-O-glukoside. Triplicate measurements were taken, and data were presented as mean \pm standard deviation $(\mathrm{SD})$.

\section{Determination of total polyphenols, flavonoids, anthocyanins and antioxidant activity}

The content of total polyphenols was determined according to the Folin-Ciocalteu procedure [21-23] using gallic acid as standard and expressing the results as gallic acid equivalents (GAE) per gram of jam samples (mg GAE/g). Content of total flavonoid was measured by the aluminum chloride spectrophotometric method described by Zhishen et al. [24], with catechin as a standard. The results were expressed as a gram of catechin equivalents (CE) per gram of jam samples (mg CE/g). The total anthocyanin content of the acidified methanol extracts was determined using the $\mathrm{pH}$-differential method [25] and expressed as miigrams of cyanidin-3-O-glucoside equivalents/g jam (mg cy-3-glu/g). For the DPPH method [26], which is slightly modified, a solution of DPPH $\left(1 \times 10^{-4} \mathrm{~mol} / \mathrm{L}\right)$ was prepared in methanol. $5.0 \mathrm{~mL}$ of DPPH solution were mixed with $100 \mu \mathrm{L}$ of jam extract and was filled with methanol to $10 \mathrm{~mL}$. $30 \mathrm{~min}$ after the reaction began the discoloration of the DPPH radical was measured at $520 \mathrm{~nm}$. The calibration curve (Trolox equivalent) was plotted as a function of the decrease in absorbance of DPPH radical scavenging activity. The results were given as millimoles of Trolox equivalents (TE) per gram of jam sample (mmol TE/g).

\section{Statistical analysis}

All the data are presented as the mean \pm standard deviation (SD) for triplicate determinations. Differences in the antioxidant activity and total polyphenols, flavonoids and anthocyanins content samples were tested by the Tukey's test. Statistical analysis was performed using a statistical package running on a computer (Excel Microsoft Office 365). A probability of $p<0.05$ was considered to be statistically significant [27].

\section{Results and discussions}

According to obtained results given in Table 1, total polyphenolic (TP), total flavonoid (TF), total anthocyanin (TA) contents and antioxidant activity of jam samples decreased in time and temperaturedependent manners but is more noticeable in sugar than in sugar-low jam at all applied temperatures.

Slight loss in TP, TF, TA, and antioxidant activity was observed after $5 \mathrm{~min}$ of heating with retention percentage yielding approximately 75-91, 79-87, 59-74 and 82-90\% in sugar jam and 87-97\%, 90-97\%, $85-95 \%$ and $90-94 \%$ in sugar-low jam samples at temperatures $105,100,95$ and $90^{\circ} \mathrm{C}$, respectively.

However, significant to moderate loss in TP, TF, TA, and antioxidant activity contents was noticed after 20 min of heating, with retention percentage yielding approximately 30-47, 45-57, 28-57 and 38$62 \%$ in sugar jam and 50-70, 57-76, 54-76 and 77-90\% in sugar-low jam at temperatures 105, 100, 95 and $90^{\circ} \mathrm{C}$, respectively.

The most significant decrease in TP, TF, and TA contents, and antioxidant activity, after $30 \mathrm{~m}$ of the thermal treatment at $105{ }^{\circ} \mathrm{C}$, was observed. Retention percentages yielding 26.4, 39.6, 23.9 and 31.6\% in sugar jam, and 40.4, 53.6, 50.2 and $62.8 \%$ in sugar-low jam, respectively. 
Furthermore, strong positive correlations were observed between DPPH antioxidant capacity and total phenols, flavonoids and anthocyanins $\left(R^{2}=0.9480 ; p<0.0001 ; R^{2}=0.9659 ; p<0.004 ; R^{2}=0.9835\right.$; $\mathrm{p}<0.0001)$

\section{HPLC analysis of individual anthocyanins}

The results regarding changes in the content of individual anthocyanins in sugar jam and in the sugarlow jam are represented in Table 2 and Table 3. Ten peaks were identified: delphinidin-3-O-galactoside, delphinidin-3-O-glucoside, cyanidin-3- $O$-galactoside, delphinidin-3-arabinoside, cyanidin-3-O- glucoside, petunidin-3-galactoside, cyanidin-3-O-arabinoside, peonidin-3-galactoside, petunidin-3-O-arabinoside and malvidin-3-galactoside. Glycosides of delphinidin were the most abundant, followed by glycosides of petunidin and cyanidin, while the galactosides of peonidin and malvidin were minor constituents. Preparation of jam of both formulations at $90^{\circ} \mathrm{C}$ resulted in the greatest retention of all identified individual anthocyanins.

Comparing anthocyanidins which contained galactose in jam samples which were heated at $90^{\circ} \mathrm{C}$ for $30 \mathrm{~min}$, the highest retention was observed at cyanidin-3-O-galactoside $(73.1 \%)$, followed by peonidin3 -galactoside $(68.4 \%)$, petunidin-3-galactoside $(67.8 \%)$, delphinidin-3- $O$-galactoside $(51.9 \%)$ and malvidin-3-galactoside (41.5\%) in sugar jam. The retention of galactosides in sugar-low jam was higher and followed the same order: cyanidin-3-O-galactoside $(88.0 \%)>$ peonidin-3-galactoside $(85.2 \%)>$ petunidin-3-galactoside $(79.5 \%)>$ delphinidin-3-O-galactoside $(78.6 \%)>$ malvidin-3-galactoside $(70.5 \%)$. There were two identified anthocyanidins containing glucose, with cyanidin-3-O-glucoside retaining better $(80.5 \%)$ than delphinidin-3-O-glucoside $(60.6 \%)$ in sugar jam. Also, in sugar-low jam cyanidin-3-O-glucoside retained slightly better (85.4\%) than delphinidin-3-O-glucoside (83.6\%). Among three arabinosides, the highest retention was observed at cyanidin-3-O-arabinoside, followed by petunidin-3-O-arabinoside and delphinidin-3-arabinoside and yielding $62.1 \%, 60.7 \%$ and $57.5 \%$ in sugar jam and $72.9 \%, 72.3 \%$ and $69 \%$ in sugar-low jam, respectively

\section{Table 1}

Total phenolic (TP), total flavonoid (TF), total anthocyanin (TA) contents and antioxidant activity during processing of sugar and sugar-low bilberry jams

\begin{tabular}{|c|c|c|c|c|c|c|c|c|c|}
\hline \multirow[b]{2}{*}{ Temp $\left({ }^{\circ} \mathrm{C}\right)$} & \multirow[b]{2}{*}{ Time (min) } & \multicolumn{4}{|c|}{$\mathrm{TP}$ (mg GAE/g) } & \multicolumn{4}{|c|}{$\mathrm{TF}(\mathrm{mg} \mathrm{CE} / \mathrm{g})$} \\
\hline & & Sugar jam & RSD (\%) & Low-sugar jam & $\begin{array}{l}\text { RSD } \\
(\%)\end{array}$ & Sugar jam & $\begin{array}{l}\text { RSD } \\
(\%)\end{array}$ & Low-sugar jam & $\begin{array}{l}\text { RSD } \\
(\%)\end{array}$ \\
\hline & & $4.02 \pm 0.03^{18}$ & $0.70^{*}$ & $5.29 \pm 0.01^{2 *}$ & $0.27 \underline{*}$ & $0.348 \pm 0.004^{a *}$ & $1.02 *$ & $0.399=0.003 \approx$ & 0.71 米 \\
\hline \multirow{5}{*}{90} & 5 & $3.66 \pm 0.06^{2}$ & 1.55 & $5.11=0.02^{\mathrm{a}}$ & 0.33 & $0.302 \pm 0.006^{\mathrm{sb}}$ & 1.88 & $0.388 \pm 0.001^{\mathrm{a}}$ & 0.36 \\
\hline & 10 & $2.85 \pm 0.06^{2}$ & 1.98 & $4.43 \pm 0.01^{2}$ & 0.29 & $0.262 \pm 0.004^{b}$ & 1.62 & $0.355=0.001^{\mathrm{a}}$ & 0.40 \\
\hline & 15 & $2.32 \pm 0.01^{2}$ & 0.61 & $4.02 \pm 0.04^{\mathrm{a}}$ & 1.09 & $0.227 \pm 0.001^{\mathrm{bc}}$ & 0.62 & $0.322=0.001^{\mathrm{a}}$ & 0.22 \\
\hline & 20 & $1.89 \pm 0.01^{2 b}$ & 0.75 & $3.70 \pm 0.03^{\mathrm{a}}$ & 0.91 & $0.200 \pm 0.002^{\mathrm{bed}}$ & 1.06 & $0.304=0.002^{\mathrm{a}}$ & 0.70 \\
\hline & 30 & $1.69 \pm 0.01^{2 b}$ & 0.84 & $3.28 \pm 0.04^{\mathrm{ab}}$ & 1.31 & $0.182 \pm 0.003^{\text {bed }}$ & 1.55 & $0.291=0.002 \mathrm{~s}$ & 0.73 \\
\hline \multirow{5}{*}{95} & 5 & $3.54 \pm 0.01^{\mathrm{abc}}$ & 0.20 & $5.00 \pm 0.07^{\mathrm{ab}}$ & 1.41 & $0.295 \pm 0.001^{\mathrm{bed}}$ & 0.48 & $0.383=0.002^{\mathrm{a}}$ & 0.55 \\
\hline & 10 & $2.52=0.06^{\mathrm{abc}}$ & 2.24 & $4.20=0.04^{\mathrm{ab}}$ & 0.92 & $0.251 \pm 0.001 \mathrm{bod}$ & 0.56 & $0.343=0.003 \mathrm{a}$ & 0.82 \\
\hline & 15 & $1.89 \pm 0.01^{\mathrm{abcd}}$ & 0.38 & $3.52=0.03 \mathrm{ab}$ & 0.96 & $0.205 \pm 0.003 \mathrm{bed}$ & 1.38 & $0.299=0.004^{\mathrm{a}}$ & 1.18 \\
\hline & 20 & $1.59 \pm 0.01^{\text {becd }}$ & 0.89 & $3.31=0.04^{\mathrm{ab}}$ & 1.13 & $0.182 \pm 0.001^{\mathrm{bed}}$ & 0.78 & $0.283 \pm 0.001^{\mathrm{a}}$ & 0.50 \\
\hline & 30 & $1.36 \pm 0.07^{\mathrm{sbcde}}$ & 5.20 & $3.19=0.03^{\mathrm{ab}}$ & 0.82 & $0.162 \pm 0.001^{\mathrm{bed}}$ & 0.87 & $0.270=0.001^{\mathrm{a}}$ & 0.52 \\
\hline \multirow{5}{*}{100} & 5 & $3.32 \pm 0.03$ abcda & 0.85 & $4.77 \pm 0.05 \mathrm{ab}$ & 0.95 & $0.281 \pm 0.001^{\text {bed }}$ & 0.50 & $0.374=0.003^{\mathrm{a}}$ & 0.76 \\
\hline & 10 & $2.26 \pm 0.04$ absdo & 1.88 & $3.82 \pm 0.05 \mathrm{ab}$ & 1.19 & $0.235 \pm 0.001^{\text {bed }}$ & 0.60 & $0.314=0.001^{\mathrm{a}}$ & 0.45 \\
\hline & 15 & $1.60 \pm 0.04$ bbcdo & 2.65 & $3.09 \pm 0.08^{\mathrm{abc}}$ & 2.59 & $0.191 \pm 0.003 \mathrm{kcd}$ & 1.48 & $0.273 \pm 0.001^{2}$ & 0.52 \\
\hline & 20 & $1.30 \pm 0.03$ abcdo & 2.18 & $2.95 \pm 0.05^{\mathrm{abc}}$ & 1.63 & $0.171 \pm 0.001^{\text {bed }}$ & 0.83 & $0.253=0.003=$ & 1.12 \\
\hline & 30 & $1.21 \pm 0.01$ abcdo & 1.11 & $2.24 \pm 0.04^{\mathrm{sbc}}$ & 1.97 & $0.152 \pm 0.002^{\mathrm{bod}}$ & 1.39 & $0.237 \pm 0.001^{\mathrm{a}}$ & 0.60 \\
\hline \multirow{5}{*}{105} & 5 & $3.01 \pm 0.03$ abcdo & 0.94 & $4.63 \pm 0.01^{\mathrm{abc}}$ & 0.31 & $0.275 \pm 0.002 \mathrm{bod}$ & 0.77 & $0.360=0.002^{\mathrm{a}}$ & 0.59 \\
\hline & 10 & $1.98 \pm 0.04^{\mathrm{bcds}}$ & 2.14 & $3.27 \pm 0.01^{b c}$ & 0.30 & $0.217 \pm 0.001^{\mathrm{cd}}$ & 0.65 & $0.298 \pm 0.004^{\mathrm{ab}}$ & 1.18 \\
\hline & 15 & $1.32 \pm 0.01^{\text {code }}$ & 0.53 & $2.78 \pm 0.02^{\circ}$ & 0.69 & $0.171=0.002^{\mathrm{d}}$ & 1.25 & $0.251 \pm 0.001 \mathrm{ab}$ & 0.56 \\
\hline & 20 & $1.19 \pm 0.01^{\mathrm{de}}$ & 0.60 & $2.63 \pm 0.02^{\circ}$ & 0.70 & $0.156 \pm 0.001^{\mathrm{d}}$ & 0.91 & $0.227 \pm 0.003 \mathrm{ab}$ & 1.25 \\
\hline & 30 & $1.06 \pm 0.01^{\mathrm{e}}$ & 0.60 & $2.14 \pm 0.04^{c}$ & 1.66 & $0.138 \pm 0.002^{\mathrm{d}}$ & 1.53 & $0.214 \pm 0.004^{b}$ & 1.66 \\
\hline
\end{tabular}


Table 1 (Continuated)

\begin{tabular}{|c|c|c|c|c|c|c|c|c|c|}
\hline \multirow[b]{2}{*}{ Temp $\left({ }^{\circ} \mathrm{C}\right)$} & \multirow{2}{*}{$\begin{array}{l}\text { Time } \\
(\min )\end{array}$} & \multicolumn{4}{|c|}{ TA (mg cy-3-gly/g) } & \multicolumn{4}{|c|}{ DPPH (mmol TE/g) } \\
\hline & & Sugar jam & $\begin{array}{l}\text { RSD } \\
(\%)\end{array}$ & Low-sugar jam & RSD (\%) & Sugar jam & $\begin{array}{l}\text { RSD } \\
(\%)\end{array}$ & Low-sugar jam & $\begin{array}{l}\text { RSD } \\
(\%)\end{array}$ \\
\hline & & $1.88 \pm 0.01^{2 *}$ & $0.71 *$ & $2.41 \pm 0.02^{2 *}$ & $0.94 *$ & $3.01 \pm 0.05^{*}$ & $1.83 *$ & $3.55 \pm 0.08^{2 *}$ & $2.22 *$ \\
\hline \multirow{5}{*}{90} & 5 & $1.39 \pm 0.01^{\mathrm{bb}}$ & 0.87 & $2.28 \pm 0.02^{\mathrm{ab}}$ & 1.05 & $2.71 \pm 0.03^{2}$ & 1.02 & $3.35 \pm 0.07^{\mathrm{ab}}$ & 2.13 \\
\hline & 10 & $1.26 \pm 0.01^{1 \mathrm{~b}}$ & 1.07 & $2.14 \pm 0.04^{2 \mathrm{bb}}$ & 2.01 & $2.38 \pm 0.01^{2}$ & 0.59 & $3.30 \pm 0.04 \mathrm{ab}$ & 1.24 \\
\hline & 15 & $1.16 \pm 0.02^{\mathrm{be}}$ & 1.33 & $1.98 \pm 0.02^{\mathrm{bb}}$ & 0.93 & $2.14 \pm 0.01^{2}$ & 0.56 & $3.24 \pm 0.06^{\mathrm{ab}}$ & 1.90 \\
\hline & 20 & $1.07 \pm 0.01^{\mathrm{bc}}$ & 0.99 & $1.83 \pm 0.02^{2 \mathrm{~b}}$ & 0.93 & $1.86 \pm 0.04^{2 b}$ & 2.05 & $3.18 \pm 0.02^{\mathrm{ab}}$ & 0.70 \\
\hline & 30 & $0.93 \pm 0.01^{\mathrm{bc}}$ & 1.29 & $1.63 \pm 0.05^{\mathrm{thc}}$ & 3.13 & $1.48 \pm 0.02^{\mathrm{bb}}$ & 1.29 & $3.08 \pm 0.03 \mathrm{ab}$ & 1.11 \\
\hline \multirow{5}{*}{95} & 5 & $1.33 \pm 0.01^{\mathrm{be}}$ & 1.06 & $2.21 \pm 0.06^{\mathrm{kbc}}$ & 2.59 & $2.63 \pm 0.07^{\mathrm{ab}}$ & 2.50 & $3.32 \pm 0.08^{\mathrm{ab}}$ & 2.44 \\
\hline & 10 & $1.11 \pm 0.01^{\mathrm{bc}}$ & 1.27 & $2.01 \pm 0.03^{\text {zbc }}$ & 1.66 & $2.22 \pm 0.02^{\mathrm{bb}}$ & 1.08 & $3.25 \pm 0.04 \mathrm{bb}$ & 1.17 \\
\hline & 15 & $0.98 \pm 0.02^{\mathrm{bed}}$ & 1.59 & $1.79 \pm 0.06^{\mathrm{zkcd}}$ & 3.42 & $1.99 \pm 0.04 \mathrm{bb}$ & 1.99 & $3.12 \pm 0.01 \mathrm{bb}$ & 0.37 \\
\hline & 20 & $0.86 \pm 0.02^{\mathrm{bed}}$ & 2.05 & $1.64 \pm 0.03^{\text {zecd }}$ & 1.85 & $1.65 \pm 0.02 \mathrm{bb}$ & 1.55 & $3.05 \pm 0.01 \mathrm{ab}$ & 0.32 \\
\hline & 30 & $0.72 \pm 0.01^{\mathrm{bed}}$ & 1.96 & $1.44 \pm 0.04^{\mathrm{ekcd}}$ & 2.78 & $1.26 \pm 0.02 \mathrm{zb}$ & 1.57 & $2.85 \pm 0.04 \mathrm{ab}$ & 1.46 \\
\hline \multirow{5}{*}{100} & 5 & $1.20 \pm 0.02^{\mathrm{bed}}$ & 1.53 & $2.12 \pm 0.05^{\text {tkcad }}$ & 1.40 & $2.56 \pm 0.06^{2 \mathrm{~b}}$ & 2.43 & $3.28 \pm 0.02^{\mathrm{ab}}$ & 0.60 \\
\hline & 10 & $0.99 \pm 0.02^{\mathrm{cd}}$ & 1.92 & $1.85 \pm 0.08^{\mathrm{sccd}}$ & 2.95 & $1.97 \pm 0.02^{b}$ & 1.08 & $3.18 \pm 0.03 \mathrm{ab}$ & 0.91 \\
\hline & 15 & $0.81 \pm 0.02^{\mathrm{cdo}}$ & 2.19 & $1.68 \pm 0.04^{\mathrm{ekcd}}$ & 4.84 & $1.67 \pm 0.03^{\mathrm{b}}$ & 1.87 & $3.05 \pm 0.02 \mathrm{ab}$ & 0.58 \\
\hline & 20 & $0.73 \pm 0.01^{\text {cde }}$ & 1.74 & $1.51 \pm 0.02^{\mathrm{bed}}$ & 2.62 & $1.39 \pm 0.02^{\mathrm{be}}$ & 1.26 & $2.91 \pm 0.04 \mathrm{bb}$ & 1.38 \\
\hline & 30 & $0.61 \pm 0.011^{\text {cosef }}$ & 1.05 & $1.35 \pm 0.02^{\mathrm{bcd}}$ & 1.21 & $1.11 \pm 0.06^{\mathrm{bcd}}$ & 5.10 & $2.68 \pm 0.03 \mathrm{ab}$ & 1.14 \\
\hline \multirow{5}{*}{105} & 5 & $1.11 \pm 0.01^{\text {cdef }}$ & 0.63 & $2.04 \pm 0.03^{\mathrm{bcd}}$ & 1.28 & $2.47 \pm 0.02^{\mathrm{bcd}}$ & 0.66 & $3.20 \pm 0.03 \mathrm{ab}$ & 0.82 \\
\hline & 10 & $0.84 \pm 0.01^{\text {dof }}$ & 1.43 & $1.73 \pm 0.01^{\mathrm{cd}}$ & 0.82 & $1.77 \pm 0.01^{\mathrm{bed}}$ & 0.60 & $3.08 \pm 0.05 \mathrm{ab}$ & 1.79 \\
\hline & 15 & $0.64 \pm 0.01^{\text {of }}$ & 1.54 & $1.46 \pm 0.02^{\mathrm{cd}}$ & 1.45 & $1.37 \pm 0.01^{\mathrm{cd}}$ & 0.77 & $2.92 \pm 0.03^{b}$ & 1.02 \\
\hline & 20 & $0.53 \pm 0.01^{\mathrm{f}}$ & 1.74 & $1.30 \pm 0.02^{\mathrm{d}}$ & 1.25 & $1.16 \pm 0.02^{\mathrm{d}}$ & 1.58 & $2.76 \pm 0.03^{b}$ & 1.28 \\
\hline & 30 & $0.45 \pm 0.01^{\mathrm{f}}$ & 2.51 & $1.21 \pm 0.01^{\mathrm{d}}$ & 0.82 & $0.95 \pm 0.01^{\mathrm{d}}$ & 1.20 & $2.23 \pm 0.06^{6}$ & 2.29 \\
\hline
\end{tabular}

Table 2

Content of individual anthocyanins $(\mathrm{mg} / \mathrm{kg})$ in sugar bilberry jam during processing

\begin{tabular}{|c|c|c|c|c|c|c|c|c|c|c|c|}
\hline $\begin{array}{l}\text { Temp. } \\
\left({ }^{\circ} \mathrm{C}\right)\end{array}$ & $\begin{array}{l}\text { Time } \\
(\min )\end{array}$ & $\begin{array}{l}\text { Delphinidin-3-O- } \\
\text { galactoside }\end{array}$ & $\begin{array}{l}\text { RSD } \\
(\%)\end{array}$ & $\begin{array}{l}\text { Delphinidin-3-O- } \\
\text { glucoside }\end{array}$ & $\begin{array}{l}\text { RSD } \\
(\%)\end{array}$ & $\begin{array}{l}\text { Cyanidin-3- } \\
\text { O-galactoside }\end{array}$ & $\begin{array}{l}\mathrm{RSD} \\
(\%)\end{array}$ & $\begin{array}{l}\text { Delphinidin-3- } \\
\text { arabinoside }\end{array}$ & $\begin{array}{l}\text { RSD } \\
(\%)\end{array}$ & $\begin{array}{l}\text { Cyanidin-3-O- } \\
\text { glucoside }\end{array}$ & $\begin{array}{l}\text { RSD } \\
(\%)\end{array}$ \\
\hline & & $177 \pm 7^{28}$ & $4.01^{*}$ & $198 \pm 4^{2 *}$ & $2.14^{8}$ & $67 \pm 1^{2 *}$ & $2.10^{8}$ & $32.7 \pm 0.7^{*}$ & $2.21^{*}$ & $118 \pm 1^{\mathrm{a} *}$ & $1.20^{*}$ \\
\hline \multirow{5}{*}{90} & 5 & $127 \pm 6^{2 b}$ & 4.46 & $133 \pm 7^{\mathrm{a}}$ & 5.31 & $62 \pm 2^{\mathrm{a}}$ & 3.44 & $24.7 \pm 0.9$ & 3.49 & $100 \pm 1^{\mathrm{ab}}$ & 1.42 \\
\hline & 10 & $110 \pm 4^{\mathrm{abc}}$ & 3.87 & $132 \pm 4^{\mathrm{a}}$ & 3.21 & $61=1^{\mathrm{a}}$ & 2.08 & $22.5=0.8^{\mathrm{a}}$ & 3.76 & $99 \pm 1^{\mathrm{ab}}$ & 1.15 \\
\hline & 15 & $98 \pm 7^{\mathrm{abcd}}$ & 7.18 & $130 \pm 8^{\mathrm{ab}}$ & 6.55 & $59=1^{2}$ & 2.27 & $21.9=0.6^{\mathrm{a}}$ & 2.91 & $98 \pm 4^{\mathrm{ab}}$ & 4.34 \\
\hline & 20 & $95 \pm 1^{\text {bede }}$ & 1.48 & $122 \pm 3^{\mathrm{abc}}$ & 2.33 & $56 \pm 1^{2}$ & 1.78 & $20.0 \pm 0.8^{\mathrm{a}}$ & 4.24 & $96 \pm 1 \mathrm{ab}$ & 1.48 \\
\hline & 30 & $92 \pm 4^{\circ .56}$ & 4.63 & $120 \pm 1^{\mathrm{ab} b d}$ & 1.18 & $49=2^{\mathrm{a}}$ & 4.02 & $18.8 \pm 0.9$ & 4.59 & $95 \pm 1^{\mathrm{abc}}$ & 1.04 \\
\hline \multirow{5}{*}{95} & 5 & $55 \pm 1^{0.05 e}$ & 2.57 & $75 \pm 3^{\mathrm{abcd}}$ & 3.80 & $27=1^{\mathrm{ab}}$ & 2.83 & $9.4 \pm 0.7^{\mathrm{b}}$ & 7.52 & $44 \pm 1^{\mathrm{abc}}$ & 2.59 \\
\hline & 10 & $51=3050$ & 5.50 & $64 \pm 4^{\mathrm{ab} c d}$ & 6.69 & $25 \pm 1^{\mathrm{ab}}$ & 0.58 & $8.9 \pm 0.4^{b}$ & 4.91 & $42 \pm 1^{\mathrm{abc}}$ & 2.35 \\
\hline & 15 & $50 \pm 3^{\circ}$ & 5.61 & $63 \pm 3^{60 \mathrm{~d}}$ & 4.48 & $24=0^{2 b}$ & 1.17 & $8.7 \pm 0.3^{b}$ & 3.25 & $41 \pm 1^{\mathrm{abc}}$ & 2.73 \\
\hline & 20 & $47 \pm 3$ cose & 6.36 & $55 \pm 1^{\text {bod }}$ & 2.56 & $23=1^{\mathrm{ab}}$ & 2.94 & $7.9 \pm 0.4^{b}$ & 5.20 & $39 \pm 1^{\mathrm{abc}}$ & 2.90 \\
\hline & 30 & $43 \pm 1^{\mathrm{de}}$ & 3.26 & $53 \pm 2^{60 \mathrm{cos}}$ & 4.00 & $21=1^{\mathrm{ab}}$ & 4.71 & $7.4 \pm 0.2^{\mathrm{b}}$ & 3.25 & $38 \pm 1^{\mathrm{abc}}$ & 3.73 \\
\hline \multirow{5}{*}{100} & 5 & $50=1^{\mathrm{de}}$ & 2.85 & $70 \pm 1^{60 d}$ & 1.82 & $24 \pm 1^{\mathrm{ab}}$ & 5.93 & $9.2 \pm 0.3^{\mathrm{b}}$ & 3.22 & $42 \pm 2^{\mathrm{abc}}$ & 5.07 \\
\hline & 10 & $42 \pm 1^{\circ}$ & 3.37 & $62 \pm 1^{\text {cd }}$ & 2.17 & $19 \pm 0^{\mathrm{b}}$ & 0.75 & $7.2 \pm 0.1^{\mathrm{b}}$ & 1.96 & $40 \pm 1^{\mathrm{abc}}$ & 3.17 \\
\hline & 15 & $39=3^{\circ}$ & 7.27 & $55 \pm 1^{\text {od }}$ & 1.80 & $17 \pm 1^{\mathrm{bc}}$ & 4.26 & $6.9 \pm 0.4^{b}$ & 5.12 & $34 \pm 1^{\mathrm{bbc}}$ & 3.91 \\
\hline & 20 & $33=2^{\circ}$ & 5.93 & $48 \pm 1^{\text {cd }}$ & 2.97 & $14=1^{b c}$ & 3.08 & $5.4=0.4^{b c}$ & 7.86 & $32 \pm 1^{\mathrm{abc}}$ & 3.08 \\
\hline & 30 & $23=1^{\circ}$ & 3.05 & $39 \pm 1^{\text {cd }}$ & 3.59 & $12=1^{\mathrm{bc}}$ & 1.15 & $4.4=0.1^{\mathrm{bc}}$ & 3.21 & $28 \pm 1^{\mathrm{abc}}$ & 7.04 \\
\hline \multirow{5}{*}{105} & 5 & $47 \pm 1^{\circ}$ & 3.00 & $58 \pm 1^{\mathrm{cd}}$ & 2.46 & $23=1^{b c}$ & 3.12 & $8.8=0.3^{b c}$ & 3.21 & $31 \pm 1^{\mathrm{abc}}$ & 1.39 \\
\hline & 10 & $40 \pm 3^{\circ}$ & 7.02 & $43 \pm 3$ cd & 6.56 & $19 \pm 1^{b c}$ & 5.28 & $8.3 \pm 0.3^{\mathrm{bc}}$ & 3.42 & $30 \pm 1^{\mathrm{bc}}$ & 4.73 \\
\hline & 15 & $31=1^{\circ}$ & 4.09 & $42 \pm 1^{\text {cd }}$ & 3.33 & $16 \pm 1^{b c}$ & 3.01 & $7.1=0.1^{\mathrm{bc}}$ & 1.99 & $26 \pm 1^{\circ}$ & 3.35 \\
\hline & 20 & $26 \pm 2^{\circ}$ & 6.62 & $38 \pm 3^{\text {cd }}$ & 7.45 & $14=1^{b c}$ & 4.91 & $5.7 \pm 0.1^{\mathrm{bc}}$ & 2.50 & $25 \pm 1^{\mathrm{c}}$ & 4.99 \\
\hline & 30 & $23=1^{\circ}$ & 6.23 & $35 \pm 1^{\mathrm{d}}$ & 4.05 & $12=0^{\circ}$ & 1.14 & $3.0 \pm 0.1^{c}$ & 4.70 & $22 \pm 1^{\circ}$ & 4.47 \\
\hline
\end{tabular}


Table 2 (Continuated)

\begin{tabular}{|c|c|c|c|c|c|c|c|c|c|c|c|}
\hline $\begin{array}{l}\text { Temp. } \\
\left({ }^{\circ} \mathrm{C}\right)\end{array}$ & $\begin{array}{l}\text { Time } \\
(\min )\end{array}$ & $\begin{array}{l}\text { Petunidin-3- } \\
\text { galactoside }\end{array}$ & $\begin{array}{l}\text { RSD } \\
(\%)\end{array}$ & $\begin{array}{c}\text { Cyanidin-3-O- } \\
\text { arabinoside }\end{array}$ & $\begin{array}{l}\text { RSD } \\
(\%)\end{array}$ & $\begin{array}{l}\text { Peonidin-3- } \\
\text { galactoside }\end{array}$ & $\begin{array}{l}\text { RSD } \\
(\%)\end{array}$ & $\begin{array}{l}\text { Petunidin-3-O- } \\
\text { arabinoside }\end{array}$ & $\begin{array}{l}\text { RSD } \\
(\%)\end{array}$ & $\begin{array}{l}\text { Malvidin-3- } \\
\text { galactoside }\end{array}$ & $\begin{array}{l}\text { RSD } \\
(\%)\end{array}$ \\
\hline & & $121 \pm 4^{3 *}$ & $3.50 *$ & $25.1 \pm 0.7_{-}^{\text {*⿻丷木 }}$ & $2.87^{\text {s+ }}$ & $46.6 \pm 0.6^{\mathrm{a} \text { *社 }}$ & $1.24^{2 x}$ & $107 \pm 2_{-}^{\text {a } x+40}$ & $1.72^{5 *}$ & 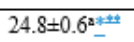 & $2.34^{\text {कx }}$ \\
\hline \multirow{5}{*}{90} & 5 & $104 \pm 7^{\mathrm{ab}}$ & 6.83 & $19.2 \pm 0.9^{\mathrm{a}}$ & 4.49 & $44.8 \pm 0.4^{2 b}$ & 0.98 & $100 \pm 2^{a}$ & 1.69 & $21.0 \pm 0.4^{\mathrm{a}}$ & 2.09 \\
\hline & 10 & $100 \pm 4^{\mathrm{ab}}$ & 4.26 & $18.8 \pm 0.8^{2}$ & 4.51 & $44.1 \pm 0.9^{\mathrm{sb}}$ & 1.96 & $93 \pm 1^{\mathrm{ab}}$ & 1.06 & $18.7 \pm 0.5^{\mathrm{a}}$ & 2.72 \\
\hline & 15 & $99 \pm 6^{\mathrm{b}}$ & 5.72 & $17.7 \pm 0.6^{2}$ & 3.59 & $43.2=0.6^{\mathrm{b}}$ & $1.47^{-}$ & $82 \pm 2^{\mathrm{ab}}$ & 2.25 & $16.0 \pm 0.6^{\mathrm{a}}$ & 3.97 \\
\hline & 20 & $96 \pm 3^{\mathrm{bc}}$ & 2.94 & $16.0 \pm 0.4^{2 b}$ & $2.39^{-}$ & $38.5 \pm 0.7^{\mathrm{b}}$ & 1.73 & $77 \pm 1^{b}$ & 1.84 & $14.1 \pm 0.4^{\mathrm{a}}$ & 2.71 \\
\hline & 30 & $82 \pm 1^{\text {bed }}$ & 1.72 & $15.6 \pm 0.9^{b}$ & 5.53 & $31.9=0.9^{b}$ & 2.71 & $65 \pm 1^{b c}$ & 1.08 & $10.3 \pm 0.6^{\mathrm{a}}$ & 5.61 \\
\hline \multirow{5}{*}{95} & 5 & $42 \pm 3^{\mathrm{cd}}$ & 6.76 & $6.4 \pm 0.2^{\circ}$ & 3.32 & $12.8 \pm 0.7^{\mathrm{kc}}$ & 5.52 & $33 \pm 1^{\mathrm{bc}}$ & $1.70^{-}$ & $6.6 \pm 0.2^{\mathrm{b}}$ & 3.23 \\
\hline & 10 & $41 \pm 2^{\mathrm{cde}}$ & 5.16 & $5.3 \pm 0.2^{c}$ & 2.95 & $12.7 \pm 0.4^{\mathrm{bc}}$ & 3.45 & $32 \pm 1^{b c}$ & 3.06 & $6.2 \pm 0.2^{\mathrm{b}}$ & 2.52 \\
\hline & 15 & $40 \pm 3^{\mathrm{de}}$ & 6.83 & $4.4 \pm 0.3^{\mathrm{d}}$ & 6.43 & $12.2 \pm 0.3^{\mathrm{c}}$ & 2.44 & $31 \pm 1^{b c}$ & 2.67 & $5.5 \pm 0.3^{\mathrm{bc}}$ & 5.14 \\
\hline & 20 & $38 \pm 1^{\mathrm{de}}$ & 3.77 & $4.0 \pm 0.2^{\mathrm{cd}}$ & $3.89^{\circ}$ & $11.8 \pm 0.4^{c}$ & 3.48 & $29 \pm 1^{\mathrm{bed}}$ & 3.96 & $5.1 \pm 0.2^{\mathrm{bc}}$ & 3.04 \\
\hline & 30 & $34 \pm 2^{\mathrm{de}}$ & 6.20 & $3.9 \pm 0.2^{\mathrm{cd}}$ & 6.16 & $11.5 \pm 0.2^{c}$ & 2.09 & $27 \pm 1^{\text {bedo }}$ & 5.18 & $4.6 \pm 0.2^{\mathrm{bod}}$ & $5.23^{-}$ \\
\hline \multirow{5}{*}{100} & 5 & $40 \pm 1^{\mathrm{de}}$ & 3.14 & $3.8=0.2^{\mathrm{cd}}$ & 4.10 & $12.0 \pm 0.3^{\mathrm{c}}$ & $2.47^{-}$ & $33 \pm 1^{\text {bede }}$ & 2.15 & $6.1 \pm 0.2^{\mathrm{bed}}$ & 2.57 \\
\hline & 10 & $33 \pm 1^{\mathrm{de}}$ & 4.07 & $4.0 \pm 0.1^{\mathrm{cd}}$ & 3.54 & $10.8 \pm 0.1^{c}$ & 1.31 & $29 \pm 1^{\text {bede }}$ & 4.37 & $4.7 \pm 0.1^{\mathrm{bos}}$ & 3.02 \\
\hline & 15 & $27 \pm 1^{\mathrm{d} \theta}$ & 3.64 & $3.4 \pm 0.2^{\mathrm{cd}}$ & 6.31 & $9.3 \pm 0.4^{c}$ & 3.82 & $25 \pm 1^{b e d \theta}$ & 1.41 & $4.0 \pm 0.2^{6 \mathrm{cos}}$ & 5.29 \\
\hline & 20 & $24 \pm 1^{\mathrm{do}}$ & 5.89 & $2.7 \pm 0.2^{\mathrm{d}}$ & 6.81 & $7.4 \pm 0.4^{c}$ & 5.73 & $21 \pm 1^{b e d e}$ & 4.63 & $3.4 \pm 0.2^{\mathrm{bed}}$ & 5.49 \\
\hline & 30 & $19=1^{\text {def }}$ & 7.39 & $2.1 \pm 0.1^{\mathrm{d}}$ & 6.87 & $6.9 \pm 0.1^{c}$ & 2.05 & $18 \pm 1^{\mathrm{b} d \mathrm{de}}$ & 3.15 & $2.8 \pm 0.1^{\mathrm{bede}}$ & 5.03 \\
\hline \multirow{5}{*}{105} & 5 & $41=1^{\text {dof }}$ & 3.47 & $3.9 \pm 0.2^{\mathrm{d}}$ & 5.38 & $10.6 \pm 0.7^{\mathrm{as}}$ & 6.68 & $28 \pm 1^{\mathrm{da} \theta}$ & 2.57 & $5.9 \pm 0.2^{\mathrm{bedo}}$ & $3.60^{\circ}$ \\
\hline & 10 & $26 \pm 1^{\mathrm{def}}$ & 2.76 & $3.7 \pm 0.2^{\mathrm{d}}$ & $4.61^{-}$ & $9.8 \pm 0.2^{\mathrm{cs}}$ & 2.45 & $25 \pm 1^{\mathrm{do}}$ & 2.86 & $4.6 \pm 0.2^{\mathrm{cde}}$ & 3.71 \\
\hline & 15 & $22 \pm 1^{\mathrm{dof}}$ & 6.31 & $3.4 \pm 0.2^{\mathrm{d}}$ & 5.82 & $8.4 \pm 0.5^{\mathrm{cd}}$ & 5.76 & $22 \pm 1^{\circ}$ & 3.35 & $4.2 \pm 0.2^{\mathrm{de}}$ & 4.67 \\
\hline & 20 & $19=0^{\text {of }}$ & 1.50 & $3.2 \pm 0.1^{\mathrm{d}}$ & 4.01 & $8.0 \pm 0.1^{\mathrm{cd}}$ & 1.77 & $16 \pm 1$ & 4.25 & $3.7 \pm 0.1^{\mathrm{de}}$ & 3.48 \\
\hline & 30 & $17 \pm 1^{f}$ & 4.17 & $2.8 \pm 0.1^{\mathrm{d}}$ & 3.61 & $7.0=0.1^{\mathrm{d}}$ & 2.03 & $11 \pm 1$ & 4.65 & $2.7 \pm 0.1^{\circ}$ & 3.72 \\
\hline
\end{tabular}

Values with different letters within columns are statistically different at $\mathrm{p}<0.05$ using Tukey's test

Table 3

Content of individual anthocyanins ( $\mathrm{mg} / \mathrm{kg}$ ) in sugar-low bilberry jam during processing

\begin{tabular}{|c|c|c|c|c|c|c|c|c|c|c|c|}
\hline $\begin{array}{l}\text { Temp. } \\
\left({ }^{\circ} \mathrm{C}\right)\end{array}$ & $\begin{array}{l}\text { Time } \\
(\min )\end{array}$ & $\begin{array}{l}\text { Delphinidin-3-O- } \\
\text { galactoside }\end{array}$ & $\begin{array}{l}\text { RSD } \\
(\%)\end{array}$ & $\begin{array}{l}\text { Delphinidin-O-3- } \\
\text { glucoside }\end{array}$ & $\begin{array}{l}\text { RSD } \\
(\%)\end{array}$ & $\begin{array}{l}\text { Cyanidin-3-O- } \\
\text { galactoside }\end{array}$ & $\begin{array}{l}\text { RSD } \\
(\%)\end{array}$ & $\begin{array}{l}\text { Delphinidin-3- } \\
\text { arabinoside }\end{array}$ & $\begin{array}{l}\text { RSD } \\
(\%)\end{array}$ & $\begin{array}{l}\text { Cyanidin-3-O- } \\
\text { glucoside }\end{array}$ & $\begin{array}{l}\text { RSD } \\
(\%)\end{array}$ \\
\hline & & $173 \pm 7^{28}$ & 4.09 하 & $195 \pm 4^{2 *}$ & $2.18^{*}$ & $100 \pm 1^{28}$ & $1.41^{*}$ & $40.0 \pm 0.6^{a *}$ & $1.45 *$ & $158 \pm 1^{\mathrm{a} *}$ & $0.90 \div$ \\
\hline \multirow{5}{*}{90} & 5 & $159 \pm 4^{2}$ & 2.67 & $185 \pm 7^{\mathrm{ab}}$ & 3.82 & $99 \pm 2^{2}$ & 2.13 & $37.5 \pm 0.9^{\mathrm{sb}}$ & 2.30 & $156 \pm 1^{\mathrm{a}}$ & 0.91 \\
\hline & 10 & $154 \pm 3^{2}$ & 1.93 & $179 \pm 4^{2 \mathrm{~b}}$ & 2.36 & $98 \pm 1^{2}$ & $1.29^{-}$ & $35.3 \pm 0.5^{\mathrm{b}}$ & 1.44 & $154 \pm 1^{\mathrm{a}}$ & 0.73 \\
\hline & 15 & $145 \pm 7^{2 b}$ & $4.88^{-}$ & $174 \pm 5^{\mathrm{ab}}$ & 2.60 & $96 \pm 1^{2}$ & $1.40^{--}$ & $32.4 \pm 0.6^{b c}$ & 1.96 & $148 \pm 4^{\mathrm{a}}$ & 2.86 \\
\hline & 20 & $141=1^{\mathrm{abc}}$ & $1.00^{\circ}$ & $171 \pm 3^{\mathrm{abc}}$ & 1.65 & $92 \pm 1^{2}$ & 1.08 & $30.9 \pm 0.7^{b c}$ & $2.15^{-}$ & $144 \pm 1^{\mathrm{a}}$ & 0.98 \\
\hline & 30 & $136 \pm 4^{\mathrm{abe}}$ & $2.60^{-}$ & $163 \pm 2^{\mathrm{abc}}$ & 1.39 & $88 \pm 2^{2}$ & 2.24 & $27.6 \pm 0.9^{\mathrm{bed}}$ & $3.13^{-1}$ & $135 \pm 1^{\mathrm{a}}$ & 0.74 \\
\hline \multirow{5}{*}{95} & 5 & $146 \pm 1^{\mathrm{abe}}$ & 0.97 & $178 \pm 3^{\mathrm{abc}}$ & 1.59 & $97 \pm 1^{2 b}$ & 0.73 & $36.9 \pm 0.5^{\mathrm{bed}}$ & 1.34 & $155 \pm 1^{\mathrm{a}}$ & 0.73 \\
\hline & 10 & $142 \pm 4^{\mathrm{abc}}$ & 2.99 & $169 \pm 4^{\mathrm{abc}}$ & 2.52 & $90 \pm 1^{2 b}$ & 1.56 & $34.1 \pm 0.4^{\mathrm{bcd}}$ & 1.29 & $142 \pm 1^{\mathrm{a}}$ & 0.70 \\
\hline & 15 & $130 \pm 3^{\mathrm{abe}}$ & 2.18 & $152 \pm 2^{\mathrm{bc}}$ & 1.40 & $81 \pm 1^{2 b}$ & $0.91^{-1}$ & $33.5 \pm 0.5^{\mathrm{ed}}$ & 1.35 & $126 \pm 1^{\mathrm{a}}$ & 0.90 \\
\hline & 20 & $128 \pm 4^{\mathrm{abe}}$ & 3.34 & $146 \pm 3^{\circ}$ & 2.04 & $79 \pm 1^{2 b}$ & 1.60 & $31.0 \pm 0.7^{\mathrm{ed}}$ & 2.24 & $123 \pm 1^{\mathrm{a}}$ & 0.92 \\
\hline & 30 & $127 \pm 2^{\mathrm{abc}}$ & 1.23 & $139 \pm 2^{c}$ & $1.53^{-}$ & $75 \pm 1^{2 b}$ & 1.32 & $29.6 \pm 0.2^{\mathrm{cd}}$ & 0.81 & $117 \pm 1^{\mathrm{a}}$ & -1.20 \\
\hline \multirow{5}{*}{100} & 5 & $131=1^{\mathrm{abc}}$ & 1.08 & $121=1^{\circ}$ & 1.05 & $70 \pm 1^{2 b}$ & 2.02 & $25.3 \pm 0.3^{\mathrm{d}}$ & 1.17 & $100 \pm 2^{\mathrm{a}}$ & 2.13 \\
\hline & 10 & $120 \pm 1^{b c}$ & 1.18 & $116 \pm 1^{\circ}$ & 1.16 & $63 \pm 1^{b}$ & 1.59 & $23.8 \pm 0.6^{\circ f}$ & 2.44 & $95 \pm 1^{\mathrm{ab}}$ & 1.34 \\
\hline & 15 & $92 \pm 3^{\text {bc }}$ & 3.07 & $106 \pm 1^{\circ}$ & 0.93 & $58 \pm 1^{\mathrm{b}}$ & 1.96 & $22.8 \pm 0.4^{\text {of }}$ & 1.55 & $90 \pm 1^{\mathrm{sb}}$ & 1.49 \\
\hline & 20 & $90 \pm 2^{b c}$ & -2.20 & $100 \pm 1^{\mathrm{dd}}$ & $1.41^{-}$ & $54 \pm 1^{\mathrm{b}}$ & $1.39^{--}$ & $20.7 \pm 0.5^{\circ f}$ & 2.25 & $89 \pm 1^{\mathrm{sbc}}$ & 1.11 \\
\hline & 30 & $86 \pm 2^{b c}$ & 2.48 & $91 \pm 1^{\text {cd }}$ & 1.55 & $51 \pm 1^{b}$ & 1.42 & $18.2 \pm 0.1^{\text {ofg }}$ & 0.78 & $88 \pm 2^{\mathrm{sbc}}$ & $2.24^{-}$ \\
\hline \multirow{5}{*}{105} & 5 & $100 \pm 1^{b c}$ & 1.41 & $117 \pm 2^{\mathrm{cd}}$ & $1.81^{-}$ & $68 \pm 1^{b}$ & 1.05 & $23.2 \pm 0.7^{\text {ofg }}$ & 3.05 & $92 \pm 1^{\mathrm{bbc}}$ & 0.77 \\
\hline & 10 & $94 \pm 2^{\circ}$ & 1.81 & $108 \pm 3$ & 2.62 & $53 \pm 1^{\mathrm{b}}$ & 1.59 & $21.5 \pm 0.3^{\mathrm{fg}}$ & 1.45 & $87 \pm 1^{\mathrm{abc}}$ & 1.62 \\
\hline & 15 & $88 \pm 1^{\circ}$ & 1.44 & $98 \pm 1^{\text {cd }}$ & $1.45^{-}$ & $50 \pm 1^{\mathrm{b}}$ & 1.15 & $19.2 \pm 0.6 \mathrm{~s}$ & 3.10 & $82 \pm 0^{\mathrm{bc}}$ & 0.35 \\
\hline & 20 & $85 \pm 2^{\circ}$ & 2.00 & $96 \pm 3^{\text {cd }}$ & 2.96 & $49 \pm 1^{b c}$ & 1.45 & $18.1 \pm 0.2 \mathrm{~s}$ & 1.09 & $80 \pm 1^{\mathrm{bc}}$ & -1.58 \\
\hline & 30 & $75 \pm 1^{\circ}$ & 1.88 & $91 \pm 1^{\mathrm{d}}$ & 1.56 & $48 \pm 1^{\circ}$ & 1.20 & $17.4 \pm 0.1 \mathrm{~g}$ & 0.81 & $78 \pm 1^{c}$ & 1.26 \\
\hline
\end{tabular}




\section{Table 3 (Continuated)}

\begin{tabular}{|c|c|c|c|c|c|c|c|c|c|c|c|}
\hline $\begin{array}{l}\text { Temp. } \\
\left({ }^{\circ} \mathrm{C}\right)\end{array}$ & $\begin{array}{l}\text { Time } \\
(\min )\end{array}$ & $\begin{array}{l}\text { Petunidin-3- } \\
\text { galactoside }\end{array}$ & $\begin{array}{l}\text { RSD } \\
(\%)\end{array}$ & $\begin{array}{l}\text { Cyanidin-3-O- } \\
\text { arabinoside }\end{array}$ & $\begin{array}{l}\text { RSD } \\
(\%)\end{array}$ & $\begin{array}{l}\text { Peonidin-3- } \\
\text { galactoside }\end{array}$ & $\begin{array}{l}\text { RSD } \\
(\%)\end{array}$ & $\begin{array}{l}\text { Petunidin-3- } \\
O \text {-arabinoside }\end{array}$ & $\begin{array}{l}\text { RSD } \\
(\%)\end{array}$ & $\begin{array}{l}\text { Malvidin-3- } \\
\text { galactoside }\end{array}$ & $\begin{array}{l}\text { RSD } \\
(\%)\end{array}$ \\
\hline & & $171 \pm 5^{a *}$ & 3.05 & $31.4 \pm 0.4_{-}^{*}$ & 1.40 & $79.7 \pm 0.9_{-}^{2 x}$ & 1.08 & $170 \pm 5_{-}^{*}$ & 2.75 & $44.1 \pm 0.7_{-}^{*}$ & 1.64 \\
\hline \multirow{5}{*}{90} & 5 & $168 \pm 6^{\mathrm{ab}}$ & 3.79 & $27.1 \pm 0.3^{2}$ & 1.09 & $77.2 \pm 0.4^{2}$ & 0.57 & $161 \pm 5^{2}$ & 2.82 & $37.9 \pm 0.4^{\mathrm{a}}$ & 1.16 \\
\hline & 10 & $166 \pm 4^{\mathrm{ab}}$ & 2.55 & $25.8 \pm 0.7^{\mathrm{2}}$ & 2.52 & $74.8 \pm 0.9^{2}$ & 1.15 & $155 \pm 4^{2 \mathrm{~b}}$ & 2.55 & $37.0 \pm 0.3^{\mathrm{a}}$ & 0.92 \\
\hline & 15 & $161 \pm 6^{\mathrm{ab}}$ & 3.52 & $23.9 \pm 0.8^{2}$ & 3.26 & $72.3 \pm 0.8^{2}$ & 1.08 & $149=3^{2 b}$ & 2.19 & $35.1 \pm 0.6^{\mathrm{a}}$ & 1.81 \\
\hline & 20 & $157 \pm 3^{\mathrm{ab}}$ & 1.80 & $23.5 \pm 0.4^{2}$ & 1.62 & $72.0 \pm 0.8^{2}$ & 1.12 & $140 \pm 1^{\mathrm{bbc}}$ & 1.01 & $33.4 \pm 0.8^{\mathrm{ab}}$ & 2.41 \\
\hline & 30 & $136 \pm 4^{\mathrm{ab}}$ & 2.80 & $22.9 \pm 0.9^{\mathrm{z}}$ & 3.77 & $67.9 \pm 0.9^{2}$ & 1.27 & $123 \pm 4^{\mathrm{abc}-}$ & 2.85 & $31.1 \pm 0.4 \mathrm{ab}$ & 1.41 \\
\hline \multirow{5}{*}{95} & 5 & $162 \pm 3^{\mathrm{ab}}$ & 1.75 & $27.9 \pm 0.4^{2}$ & 1.27 & $74.2 \pm 0.9^{2}$ & $1.24^{-}$ & $155 \pm 5^{\mathrm{abc}}$ & $3.46^{-}$ & $37.3 \pm 0.8^{\mathrm{ab}}$ & 2.09 \\
\hline & 10 & $148 \pm 2^{2 \mathrm{ecc}}$ & 1.43 & $24.6 \pm 0.7^{\mathrm{2}}$ & 2.93 & $69.5 \pm 0.6^{2}$ & 0.83 & $149 \pm 2^{\mathrm{abc}}$ & 1.62 & $36.4 \pm 0.3^{\mathrm{abc}}$ & 0.82 \\
\hline & 15 & $142 \pm 2^{2 \mathrm{bcc}}$ & 1.19 & $22.2 \pm 0.2^{\mathrm{z}}$ & 0.77 & $63.2 \pm 0.7^{\mathrm{2}}$ & $1.14^{-}$ & $140 \pm 3^{\mathrm{bc}}$ & 1.82 & $34.7 \pm 0.3 \mathrm{abc}$ & 0.82 \\
\hline & 20 & $138 \pm 4^{b c}$ & 3.17 & $21.0 \pm 0.6^{2}$ & 2.76 & $61.4 \pm 0.4^{2}$ & 0.67 & $138 \pm 4^{b c}$ & 2.88 & $34.1 \pm 0.4^{\mathrm{abc}}$ & 1.29 \\
\hline & 30 & $134 \pm 2^{\mathrm{bc}}$ & 1.59 & $20.1 \pm 0.2^{2}$ & 1.20 & $58.6 \pm 0.7^{2 b}$ & $1.13^{-}$ & $121=1^{\circ}$ & 1.17 & $30.3 \pm 0.2^{\mathrm{bc}}$ & 0.79 \\
\hline \multirow{5}{*}{100} & 5 & $104 \pm 3^{c}$ & 2.59 & $23.0 \pm 0.6^{2}$ & 2.52 & $58.3 \pm 0.6^{2 b}$ & 1.09 & $103=2^{\circ}$ & 2.07 & $28.8 \pm 0.2^{\mathrm{bc}}$ & 0.54 \\
\hline & 10 & $96 \pm 1^{c}$ & 1.40 & $21.8 \pm 0.1^{\mathrm{z}}$ & 0.65 & $47.5 \pm 0.9^{2 b}$ & 1.82 & $93 \pm 1^{\mathrm{cd}}$ & 1.37 & $22.0 \pm 0.3^{\mathrm{cd}}$ & 1.35 \\
\hline & 15 & $89 \pm 1^{\mathrm{cd}}$ & 1.11 & $19.3 \pm 0.2^{\mathrm{z}}$ & 1.10 & $45.8 \pm 0.4^{\mathrm{b}}$ & $0.77^{-}$ & $86 \pm 3^{c \mathrm{~d}}$ & 3.21 & $21.0 \pm 0.3^{\mathrm{d} \theta}$ & 1.39 \\
\hline & 20 & $87 \pm 1^{\mathrm{cd}}$ & 1.62 & $18.1 \pm 0.9^{2}$ & 4.93 & $42.2 \pm 0.4^{\mathrm{b}}$ & $1.01^{-}$ & $83 \pm 5^{\text {cde }}$ & $6.30^{--}$ & $20.4 \pm 0.2^{\mathrm{d} \mathrm{d}}$ & 0.88 \\
\hline & 30 & $86 \pm 3^{\mathrm{cd}}$ & 3.44 & $17.3 \pm 0.1^{2}$ & 0.82 & $40.2 \pm 0.1^{\mathrm{bc}}$ & 0.35 & $80 \pm 2^{\mathrm{cse}}$ & $2.46^{-}$ & $18.8 \pm 0.1^{\mathrm{do}}$ & 0.75 \\
\hline \multirow{5}{*}{105} & 5 & $94 \pm 3^{\mathrm{cd}}$ & 3.15 & $20.0 \pm 0.2^{\mathrm{z}}$ & 1.06 & $53.4 \pm 0.7^{\mathrm{bc}}$ & 1.32 & $100 \pm 4^{\text {cose }}$ & 3.53 & $21.5 \pm 0.2^{\mathrm{de}}$ & 0.99 \\
\hline & 10 & $91 \pm 2^{\mathrm{cd}}$ & 2.33 & $16.8 \pm 0.2^{\mathrm{z}}$ & 1.01 & $43.9=0.7^{\mathrm{bc}}$ & $1.51^{-}$ & $93 \pm 1^{\text {cse }}$ & 1.53 & $23.9 \pm 0.2^{\mathrm{de}}$ & 0.71 \\
\hline & 15 & $84 \pm 1^{\mathrm{d}}$ & 1.68 & $15.6 \pm 0.3^{2}$ & 2.18 & $40.0 \pm 0.5^{\circ}$ & 1.20 & $83 \pm 2^{\mathrm{de}}$ & 2.04 & $20.8 \pm 0.6^{\mathrm{de}}$ & 2.99 \\
\hline & 20 & $81 \pm 2^{\mathrm{d}}$ & 2.09 & $14.9 \pm 0.6^{2}$ & 3.90 & $38.9 \pm 0.1^{\circ}$ & 0.36 & $78 \pm 5^{\mathrm{de}}$ & $582^{-}$ & $18.6 \pm 0.6^{\circ}$ & 3.12 \\
\hline & 30 & $80 \pm 2^{\mathrm{d}}$ & 2.66 & $14.3 \pm 0.3^{2}$ & 2.08 & $36.7 \pm 0.1^{\circ}$ & $0.39^{-}$ & $76 \pm 3^{\circ}$ & $3.72^{--}$ & $17.2 \pm 0.7^{\circ}$ & 4.19 \\
\hline
\end{tabular}

Heating at $105^{\circ} \mathrm{C}$ for $30 \mathrm{~min}$ brought to severe loss in contents of all identified anthocyanins. Retention percentages of cyanidin-3-O-galactoside, peonidin-3-galactoside, petunidin-3-galactoside, delphinidin-3-O-galactoside and malvidin-3-galactoside were $17.9,15,14.0,13$ and $10.9 \%$ in sugar jam and 48.0, 46.0, 44.7, 43.3 and $39.0 \%$ in sugar-low jam, respectively.

Cyanidin-3-O-glucoside retained better with $18.6 \%$ than delphinidin-3-O-glucoside with $17.7 \%$ in sugar jam. The same was observed in a sugar-low jam where $49.4 \%$ of cyanidin-3-O-glucoside and 46.7\% of delphinidin-3-O-glucoside were retained.

The highest retention among arabinosides was observed at cyanidin-3- $O$-arabinoside, followed by petunidin-3-O-arabinoside and delphinidin-3-arabinoside and yielding $11.1,10.3$ and $9.2 \%$ in sugar jam and $45.5,44.7$ and $43.5 \%$ in a sugar-low jam.

Cyanidin-3-O-galactoside and cyanidin-3-O-glucoside were found to be the most stable, while petunidin-3- $O$-arabinoside and delphinidin-3-arabinoside were the least stable identified anthocyanins.

Our results are in agreement with a study of Trošt et al. [28] on anthocyanin degradation during storage of blueberry-aronia nectar, where the stability of individual anthocyanins with respect to aglycone was as follows: cyanidin $>$ peonidin $>$ petunidin $>$ malvidin $>$ delphinidin.

The obtained results have also shown that galactosides and glucosides retained better than arabinosides, which is in accordance with the previously mentioned study [28] where larger hexose sugars exhibit greater stability than smaller pentose sugars.

\section{Degradation kinetics of anthocyanins}

Linear regression showed that the degradation of total anthocyanins in both sugar and sugar-low jam samples followed by a first-order reaction (Figure 1 and Figure 2). In this case, a first-order reaction can generally be expressed using Eq. $1[29,30]$ where $c$ is the anthocyanin concentration at time $t, c_{o}$ is the initial concentration of total anthocyanins $(\mathrm{mg} / \mathrm{g}), t$ is the treatment time $(\mathrm{min})$ and $k$ the first-order degradation rate constant $\left(\mathrm{min}^{-1}\right)$.

$$
c=c_{0} \cdot \exp (-k \cdot t)
$$


The half-lives $\left(\mathrm{t}_{1 / 2}\right)$ of the anthocyanins were calculated by the Eq. 2 :

$$
t_{1 / 2}=-\ln (0.5) / k=0.693 / k
$$

The temperature-dependence degradation rate constant was represented by the Arrhenius, Eq. 3:

$$
k=k_{0} \times e^{-E a / R T}
$$

where $k_{o}$ is the frequency factor, $E_{a}$ is the activation energy, $R$ is the universal gas constant, and $T$ is the absolute temperature.
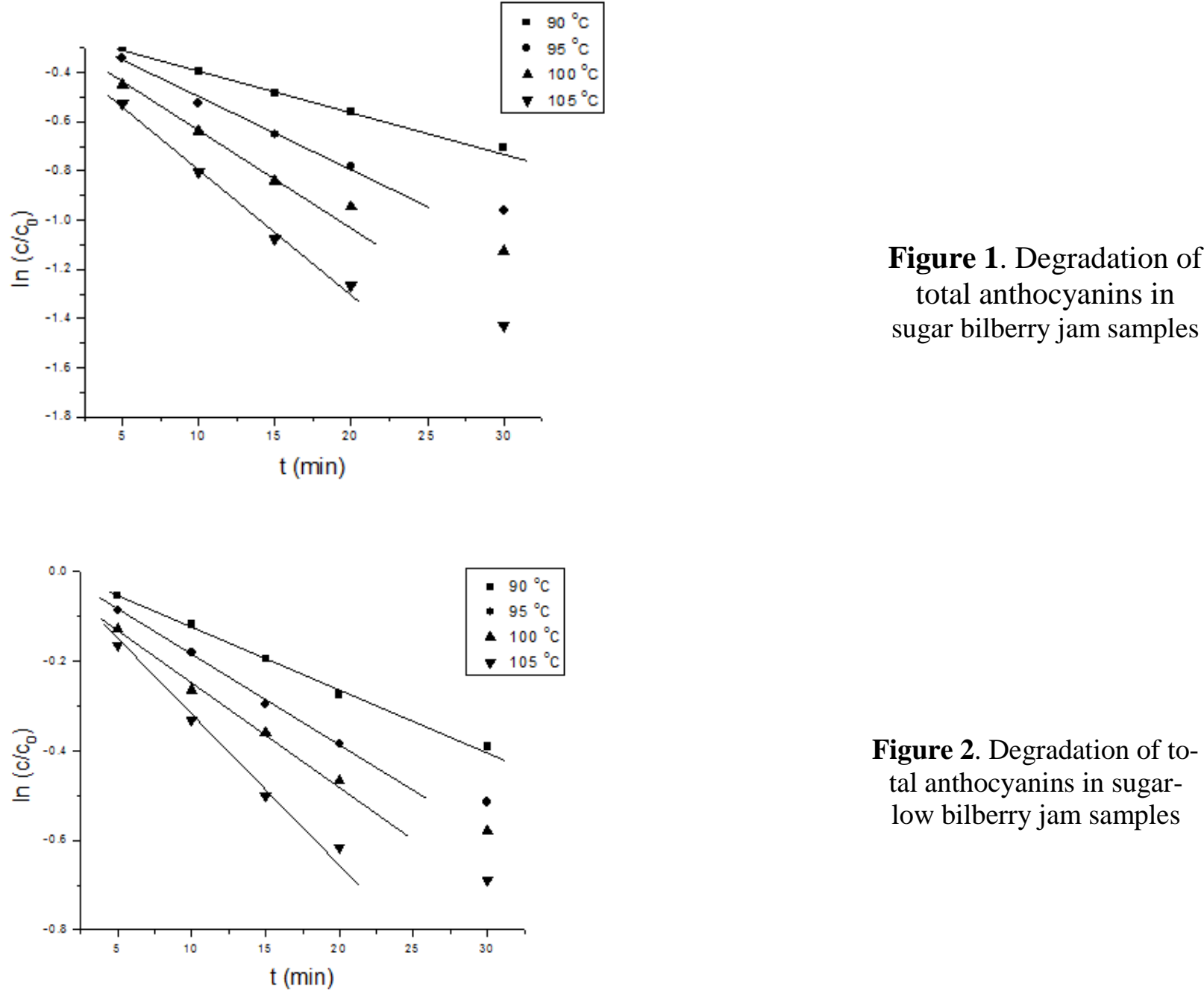

Figure 2. Degradation of total anthocyanins in sugarlow bilberry jam samples

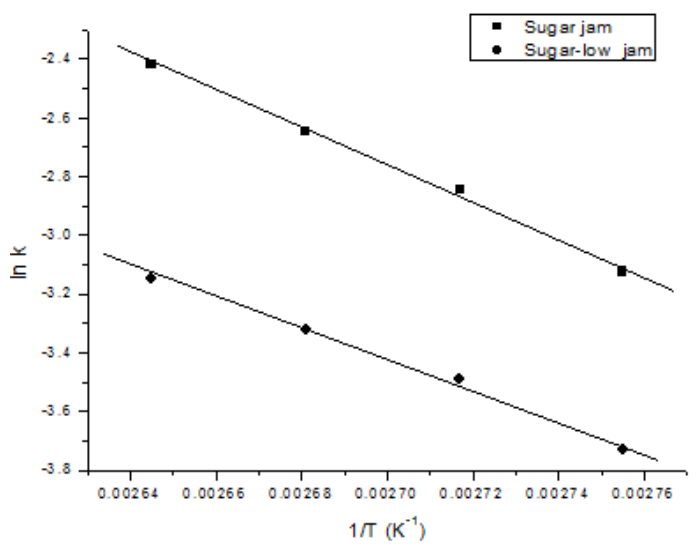

Figure 3. The Arrhenius plot for degradation of total anthocyanins in bilberry jam samples 
Effect of the heating temperature on the total anthocyanin degradation rate is shown in Figure 1 and Figure 2, and the kinetic parameters are given in Table 4. The rate constant obeyed the Arrhenius relationship (Eq. 3), and the Arrhenius plot is given in Figure 3. The high $\mathrm{R}^{2}$ values obtained from the Arrhenius plot confirm that the degradation increased with increased temperature and time $\left(0.9724<\mathrm{R}^{2}<0.9978\right)$. Therefore, the percentage of retention of total anthocyanins significantly depends on heating temperature and was ranged from $49.5 \%$ to 73.9 at $90{ }^{\circ} \mathrm{C}$, from 38.3 to $70.7 \%$ at $95^{\circ} \mathrm{C}$, from 32.4 to $63.8 \%$ at $100^{\circ} \mathrm{C}$ and from 23.9 to $59.0 \%$ at $105^{\circ} \mathrm{C}$ in sugar jam formulation. Total anthocyanins have shown greater resistance towards high temperatures exposure in the sugar-low formulation, yielding from 67.6 to $94.6 \%$ at $90^{\circ} \mathrm{C}$, from 59.7 to $91.7 \%$ at $95^{\circ} \mathrm{C}$, from 56.0 to $88.0 \%$ at $100^{\circ} \mathrm{C}$ and from 50.2 to $84.6 \%$ at $105^{\circ} \mathrm{C}$.

Table 4

Effect of heating temperature on the reaction constant rate $(\mathrm{k})$, time of half-life $\left(\mathrm{t}_{1 / 2}\right)$ and activation energy $\left(E_{a}\right)$ values of total anthocyanins degradation in sugar and sugar-low bilberry jams

\begin{tabular}{|c|c|c|c|c|}
\hline \multicolumn{5}{|c|}{ Sugar jam } \\
\hline $\begin{array}{c}\text { Temperature } \\
\left({ }^{\circ} \mathrm{C}\right)\end{array}$ & $\begin{array}{l}\mathrm{k} \times 10^{2} \\
\left(\min ^{-1}\right)\end{array}$ & $\mathrm{R}^{2}$ & $\begin{array}{c}\mathrm{t}_{1 / 2} \\
(\mathrm{~min})\end{array}$ & $\underset{(\mathrm{kJ} / \mathrm{mol})}{\mathrm{E}_{\mathrm{a}}}$ \\
\hline 90 & $4.4 \pm 0.3$ & 0.9948 & 15.7 & \multirow{4}{*}{$51 \pm 1$} \\
\hline 95 & $6.0 \pm 0.3$ & 0.9857 & 11.5 & \\
\hline 100 & $7.1 \pm 0.5$ & 0.9724 & 9.8 & \\
\hline 105 & $8.9 \pm 0.4$ & 0.9908 & 7.8 & \\
\hline \multicolumn{5}{|c|}{ Sugar-low jam } \\
\hline $\begin{array}{c}\text { Temperature } \\
\left({ }^{\circ} \mathrm{C}\right)\end{array}$ & $\begin{array}{l}\mathrm{k} \times 10^{2} \\
\left(\mathrm{~min}^{-1}\right)\end{array}$ & $\mathrm{R}^{2}$ & $\begin{array}{c}\mathrm{t}_{1 / 2} \\
(\mathrm{~min})\end{array}$ & $\underset{(\mathrm{kJ} / \mathrm{mol})}{\mathrm{E}_{\mathrm{a}}}$ \\
\hline 90 & $2.4 \pm 0.1$ & 0.9947 & 28.4 & \multirow{4}{*}{$40 \pm 1$} \\
\hline 95 & $3.2 \pm 0.2$ & 0.9978 & 21.8 & \\
\hline 100 & $3.6 \pm 0.1$ & 0.9889 & 19.1 & \\
\hline 105 & $4.3 \pm 0.2$ & 0.9904 & 16.2 & \\
\hline
\end{tabular}

The larger amount of sugar in sugar jam gave a two-fold increase in degradation rate constants and an approximately two-fold decrease in the half-lives comparing to the sugar-low formulation at all applied temperatures. The $t_{1 / 2}$ values ranged from 15.7 to $7.8 \mathrm{~min}$ for sugar jam and from 28.4 to $16.2 \mathrm{~min}$ for the sugar-low jam at $90,95,100$ and $105^{\circ} \mathrm{C}$, respectively.

The value of the activation energy was $40 \mathrm{~kJ} / \mathrm{mol}$ and $51 \mathrm{~kJ} / \mathrm{mol}$ for low-sugar and sugar jam, respectively. This finding shows that the degradation of anthocyanins is more affected by temperature elevation in sugar formulation. Obtained values are in agreement with the results of Dyrby et al. [31] on thermal degradation of anthocyanins in soft drink mediums of elderberry juice and blackcurrant pomace extracts $(56 \mathrm{~kJ} / \mathrm{mol}$ and $50 \mathrm{~kJ} / \mathrm{mol})$, but lower than the ones reported in a study of Wang and $\mathrm{Xu}$ [13] on degradation kinetics of anthocyanins in blackberry juice and concentrate $(65.06 \mathrm{~kJ} / \mathrm{mol}$ and $75.5 \mathrm{~kJ} / \mathrm{mol})$ and in a study of Kechinski et al. [14] on degradation kinetics of anthocyanins in blueberry juice (80.4 $\mathrm{kJ} / \mathrm{mol})$. 
Obtained results are in agreement with the results reported by de Moura et al. [15], where it was found that reduction in the amounts of anthocyanin compounds was greater in the sugar jam. In a study conducted by de Rosso and Mercandante [32], it was also shown that addition of sugars exhibited an adverse effect on the stability of anthocyanins from tropical fruits. In general, these results are in accordance with the pioneering work of Meschter [33], where it was illustrated the ability of some sugars and sugar degradation products to increase the rate of strawberry pigment loss. Also, thermal degradation of anthocyanins includes the chalcone formation or loss of glycosyl moieties [34] (Nayak et al., 2011). In relation to the stability, anthocyanins may suffer reactions that altered their structures due to the electronic deficiency of their flavylium nuclei [35] (Turturica et al., 2018).

\section{Conclusions}

This study showed that significant changes occur in total and individual anthocyanin contents when bilberry fruits are processed into sugar and sugar-low jams. The main practical application of this work is that it showed that larger amounts of total and individual anthocyanins are retained when jams are prepared at lower temperatures. It also showed that the presence of sugar had a negative effect on total and individual anthocyanins, as well as on total polyphenols, flavonoids and antioxidant activity of bilberry jams. According to our knowledge at this moment, this is the first work that comparatively studied degradation kinetics of anthocyanins at high temperatures in bilberry jams, immediately after preparation. Thermal degradation of bilberry anthocyanins followed first-order reaction kinetics. Higher retention of all analyzed compounds was accomplished in the sugar-low jam, in lower temperatures and shorter heating time.

Acknowledgments: This research was supported by grant number 172047 from the Serbian Ministry of Education, Science and Technological Development. The authors owe a great gratitude to Ministry for financial support.

\section{References}

1. CASTAÑEDA-OVANDO, A., PACHECO-HERNANDEZ, M.L., PAEZ-HERNANDEZ, M.E., RODRRÍGUEZ, J.A., GALAN-VIDAL, C.A., Food Chem., 113, nr. 4, 2009, p. 859

2. LOPES DA SILVA, F., ESCRIBANO-BAILON, M.T., PÉREZ ALONSO, J.J., RIVAS-GONZALO, J.C., SANTOS-BUELGA, C., LWT - Food Sci. Technol., 40, nr. 2, 2007, p. 374

3. KÄHKÖNEN, M.P., HEINÄMÄKI, J., OLLILAINEN, V., HEINONEN, M., J. Sci. Food Agric., 83, nr. 9, 2003, p. 1403

4. PANTELIDIS, G.E., VASILAKAKIS, M., MANGANARIS, G.A., DIAMANTIDIS, G., Food Chem., 102, nr. 3, 2007, p. 777

5. BOWEN-FORBES, C.S., ZHANG, Y., NAIR, M.G., J. Food Compost. Anal., 23, nr. 6, 2010, p. 554 6. CHU, W., CHEUNG, S.C.M., LAU, R.A.W., BENZIE IFF. Bilberry (Vaccinium Myrtillus L.) In I.F.F. Benzie and S. Wachtel-Galor (Eds). Herbal Medicine: Biomolecular and Clinical Aspects, 2nd ed., CRC Press/Taylor \& Francis, Boca Raton, 2011

7. SMITH, M.A.L., MARLEY, K.A., SEIGLER, D., SINGLETARY, K.W., MELINE, B., J. Food Sci., 65, nr. 2, 2000, p. 352

8. SAVIKIN, K., ZDUNIC, G., JANKOVIC, T., TASIC, S., MENKOVIC, N., STEVIC, T., DDORDEVIC, B., Plant Foods Hum. Nutr., 64, nr. 3, 2009, p. 212

9. YOU, Q., WANG, B., CHEN, F., HUANG, Z., WNANG, X., LUO, P.G., Food Chem., 125, nr. 1, 2011, p. 201

10. HEINONEN, M., Mol. Nutr. Food Res., 51, nr. 6, 2007, p. 684

11. SZAJDEK, A., BOROWSKA, E.J., Plant Foods Hum. Nutr., 63, nr. 4, 2008, p. 147

12. JIANG, T., MAO, Y, SUI, L., YANG, N., LI, S., ZHU, Z., WANG, C., YIN, S., HE, J., HE, Y., Food Chem., 274, 2019, p. 460

13. WANG, W.D., XU, S.Y., J. Food Eng., 82, nr. 3, 2007, p. 271 
14. KECHINSKI, C.P., GUIMARAES, P.V.R., NORENA, C.P.Z., TESSARO, I.C., MARCZAK, L.D., J. Food Sci., 75, nr. 2, 2010, p. 173

15. DE MOURA, S.C.S.R., DA ROCHA TAVARES, P.E., GERMER, S.P.M., NISIDA, A.L.A.C., ALVES, A,B., KANAAN, A.S., Food Bioprocess Tech., 5, nr. 6, 2012, p. 2488

16. GARCIA-VIGUERA, C., ZAFRILLA, P., ARTS ROMERO, F., ABELLAN, P., TOMAS-BARBERAN, F.A., J. Sci. Food Agric., 78, 1998, p. 565

17. HOWARD, L.R., CASTRODAlE, C., BROWNMIller, C., MAUROMOUSTAKOS, A., J. Agric.Food Chem., 58, nr. 7, 2010, p. 4022

18. MITIC, M.N., JEAN-MARC SOUQUET, J.M., OBRADOVIC, M.V., MITICC, S.S., Food Sci. Biotechnol., 21, nr. 6, 2012, p. 1619

19. NYMAN, A.N., KUMPULAINEN J.T., J. Agric. Food Chem., 49, nr. 9, 2010, p. 4183.

20. WU, X., PRIOR, R.L., J. Agric. Food Chem., 5, nr. 7, 2005, p. 2589

21. SLINKARD, K., SINGLETON, V.L., Am. J. Enol. Vitic., 28, nr. 1, 1977, p. 49

22. SINGLETON, V.L., ORHOFER, R., LAMUELA-RAVENTOS, R.M., Methods Enzymol., 299, 1999 , p. 152

23. STRATIL, P., KLEJDUS, B., KUBAN, V., J. Agric. Food Chem., 54, nr. 3, 2006, p. 607

24. ZHISHEN, J., MENGCHENG, T., WU JIANMING, W., Food Chem., 64, nr. 4, 1999, p. 555

25. GUISTI, M.M., WROLSTAD, R.E., Characterization and Measurement of Anthocyanins by UVvisible Spectroscopy, In: Wrolstad, R., Ed., Current Protocols in Food Analytical Chemistry. F1.2.1F1.2.13., John Wiley \& Sons, New York, 2001

26. BRAND-WILLIAMS, W., CUVELIER, M.E., BERSET, C., LWT - Food Sci. Technol. 28, nr. 1, 1995 , p. 25

27. MILLER, J.N. MILLER, J.C., Statistics and chemometrics for analytical chemistry, 6th ed., Pearson Education, London, 2005

28. TROST, K., GOLIC-WONDRA, A., PROSEK, M., MILIVOJEVIC, L., J. Food Sci., 73, nr. 8, 2008, p. 405

29. KIRCA, A., CEMEROGLU, B., Food Chem., 81, nr. 4, 2003, p. 583

30. HARBOURNE, N., JACQUIER, J.C., MORGAN, D.J., LYNG, J.G., Food Chem., 111, nr. 1, 2008, p. 204

31. DYRBY, M., WESTERGAARD, N., STAPELFELDT, H., Food Chem., 72, nr. 4, 2001, p. 431

32. DE ROSSO, V.V., MERCADANTE, A.Z., Innov. Food Sci. Emerg. Technol., 8, nr. 3, 2007, p. 347

33. MESCHTER, E.E., J. Agric. Food Chem., 1, nr. 8, 1953, p. 574

34. NAYAK, B., BERRIOS, J.D.J., POWERS, J., TANG, J., J. Agric. Food Chem, 59, nr 20, 2011, p. 11040

35. TURTURICA, M., STANCIUC, N., MURESAN, C., RAPEANU, G., CROITORU C., J. Food Qual., 2018, https://doi.org/10.1155/2018/1598756

$\overline{\text { Manuscript received: } 21.05 .2019}$ 